\title{
ON THE OUTER COEFFICIENT OF QUASICONFORMALITY OF A CONVEX DIHEDRAL WEDGE
}

\author{
OSSI TAARI
}

\section{Introduction}

Let $D$ and $D^{\prime}$ be domains in $R^{3}$ and $f: D \rightarrow D^{\prime}$ a homeomorphism. The numbers

$$
K_{I}(f)=\sup _{\Gamma} \frac{M(f \Gamma)}{M(\Gamma)}, \quad K_{O}(f)=\sup _{\Gamma} \frac{M(\Gamma)}{M(f \Gamma)}
$$

are called the inner and the outer dilatation of $f$. There $M(\Gamma)$ and $M(f \Gamma)$ are the moduli of the curve families $\Gamma$ and $f \Gamma$ and the suprema are taken over all families which lie in $D$. The mapping $f$ is quasiconformal if the maximal dilatation $\max \left(K_{I}(f), K_{O}(f)\right)$ is finite. Further, the inner and outer coefficients of the quasiconformality of $D$ with respect to $D^{\prime}$ are defined by the numbers

$$
K_{I}\left(D, D^{\prime}\right)=\inf _{f} K_{I}(f), \quad K_{O}\left(D, D^{\prime}\right)=\inf _{f} K_{O}(f),
$$

where $f$ runs through all homeomorphisms $f: D \rightarrow D^{\prime}$. The case when $D^{\prime}$ is a ball or a half space is of particular interest.

The problem of determining the coefficients of quasiconformality and the corresponding extremal mappings is fairly difficult and has been only solved for a few domains. In this paper we shall consider the outer coefficient problem in the case where $D$ is a convex dihedral wedge in $R^{3}$ and $D^{\prime}$ is a half space, still with certain additional local conditions for the mapping at one arbitrary edge point. Gehring and Väisälä have solved the corresponding problem for the inner coefficient [2], p. 43 (see also Väisälä [3], p. 1331). As for the theory of $n$-dimensional quasiconformal mappings we refer to [3].

\footnotetext{
${ }^{1}$ The definitions of dilatations in [2] are not exactly the same as in this paper and in [3].
} 


\section{Additional conditions}

Let $(r, \psi, \varphi)$ be spherical coordinates in $R^{3}$, where the polar angle $\varphi$ is measured from the positive half of the $x_{3}$-axis. A domain in $R^{3}$ is called a dihedral wedge of angle $\alpha, 0<\alpha \leqq 2 \pi$, if it can be mapped by means of a similarity transformation onto the domain

$$
D_{\alpha}=\{(r, \psi, \varphi) \mid r>0,0<\psi<\alpha, 0<\varphi<\pi\} .
$$

The inverse image of the $x_{3}$-axis under this mapping is said to be the edge of the dihedral wedge.

We consider now the subclass $W$ of homeomorphisms $f: \bar{D}_{\alpha} \rightarrow \bar{D}_{\pi}, 0<\alpha<\pi$, $f(0)=0$, whose restrictions $f \mid D_{\alpha}$ are quasiconformal mappings onto $D_{\pi}$ and which satisfy the following conditions $\mathrm{A}$ and $\mathrm{B}$ at the origin.

A. There is a polar angle $\varphi_{0}, 0<\varphi_{0}<\pi / 2$, such that the limit

$$
\lim _{t \rightarrow 0+} f(t e) / t=k(e) \neq 0, \infty
$$

exists for every $e \in \bar{D}_{\alpha}$ with $0 \leqq\left(e, e_{3}\right) \leqq \varphi_{0}$, where $\left(e, e_{3}\right)$ denotes the actual angle between the vectors $e$ and $e_{3}$.

Let $g_{n}: \bar{D}_{\alpha} \rightarrow \bar{D}_{\pi}$ be the sequence, defined by

$$
g_{n}(x)=n f(x / n), \quad n=1,2, \ldots
$$

We extend $g_{n}$ to a quasiconformal mapping of $R^{3}$ onto itself. First we map $D_{\alpha}$ onto $D_{\pi}$ by the folding $h, h(r, \psi, \varphi)=(r, \pi \psi / \alpha, \varphi)$. Then $g_{n} \circ h^{-1}$ will be extended to a quasi-conformal mapping $h_{1}: R^{3} \rightarrow R^{3}$ by reflection. Finally, we define a mapping $h_{2}: R^{3} \rightarrow R^{3}$ by $h_{2}(r, \psi, \varphi)=\left(r, \psi^{\prime}, \varphi\right)$, where

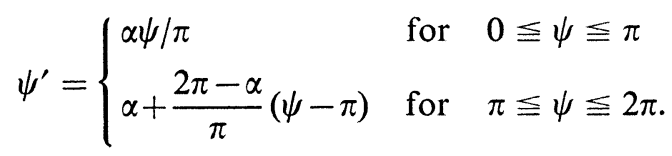

Then $\hat{g}_{n}=h_{1} \circ h_{2}^{-1}: R^{3} \rightarrow R^{3}$ is quasiconformal and $\hat{g}_{n} \mid \bar{D}_{\alpha}=g_{n}$.

Because $\left(k(e), k\left(e_{3}\right)\right)>0$ for a fixed $f$ and every $e, 0<\left(e, e_{3}\right) \leqq \varphi_{0}$ (see [1], Theorem 3.3), the sequence $\hat{g}_{n}$ is by [3], 19.4 and 20.5 a normal family and thus has a subsequence $\hat{g}_{i}, i \in J \subset N$, which converges to the limit function $\hat{g}: R^{3} \rightarrow R^{3}$ uniformly on every compact subset of $R^{3}$. By the condition $\mathrm{A} \hat{g}$ is a homeomorphism, since $\hat{g}(0)=0$ and $\hat{g}_{n}\left(e_{3}\right)=n f\left(e_{3} / n\right) \rightarrow k\left(e_{3}\right)$; see [3], 21.3. By [3], 37.2, $g=\hat{g} \mid D_{\alpha}$ is quasiconformal and

$$
K_{o}(g) \leqq \varliminf_{n \rightarrow \infty} K_{o}\left(g_{n}\right)=K_{o}(f) .
$$

The condition $\mathrm{A}$ implies that for every $e \in \bar{D}_{\alpha}, 0 \leqq\left(e, e_{3}\right) \leqq \varphi_{0}, g$ maps the ray $\{t e \mid t>0\}$ onto the ray $\{t k(e) \mid t>0\}$ linearly, $g(t e)=t k(e)$. We set the following 
additional condition for $f$ :

$$
\text { B. } \lim _{\varepsilon \rightarrow 0+}\left(\max _{\substack{\left(e, e_{3}\right)=\varepsilon \\ e \in D_{\alpha}}}\left(k(e), k\left(e_{3}\right)\right) / \min _{\substack{\left(e, e_{3}\right)=\varepsilon \\ e \in D_{\alpha}}}\left(k(e), k\left(e_{3}\right)\right)\right)=1 .
$$

This condition as well is local for $f$ at the origin. We denote

$$
K_{O}\left(D_{\alpha}, D_{\pi} ; W\right)=\inf \left\{K_{O}(f) \mid f \in W\right\}
$$

and prove the following theorem.

Theorem 1. $K_{O}\left(D_{\alpha}, D_{\pi} ; W\right)=\pi / \alpha$.

For $\varepsilon>0$ let $f \in W$ be a mapping such that

$$
K_{o}(f)<K_{o}\left(D_{\alpha}, D_{\pi} ; W\right)+\varepsilon
$$

and $g$ the limit mapping, associated with $f$ by the above process. Then $K_{o}(g)$ $\leqq K_{o}(f)$ and thus also

$$
K_{o}(g)<K_{o}\left(D_{\alpha}, D_{\pi} ; W\right)+\varepsilon .
$$

Next, to prove the Theorem 1 we consider the following curve families in $D_{\alpha}$.

\section{The curve families $\Gamma$ and $\Gamma_{1}$}

We denote

$$
D_{\alpha}\left(r_{1}, r_{2} ; \beta, \varphi_{0}\right)=\left\{(r, \psi, \varphi) \mid r_{1}<r<r_{2}, 0<\psi<\alpha, \beta<\varphi<\varphi_{0}\right\},
$$

where $r_{1}>0,0<\beta<\varphi_{0}$. Let $\Gamma$ be the family of all arcs joining the plane parts

and

$$
T_{0}=\overline{D_{\alpha}\left(r_{1}, r_{2} ; \beta, \varphi_{0}\right)} \cap\{\psi=0\}
$$

$$
\left.T_{\alpha}=\overline{D_{\alpha}\left(r_{1}, r_{2} ; \beta, \varphi_{0}\right.}\right) \cap\{\psi=\alpha\}
$$

of the boundary $\partial D_{\alpha}\left(r_{1}, r_{2} ; \beta, \varphi_{0}\right)$ in $D_{\alpha}\left(r_{1}, r_{2} ; \beta, \varphi_{0}\right)$.

Let $\varrho \in F(\Gamma)$ be arbitrary, i.e. $\int_{\gamma} \varrho d s \geqq 1$ when $\gamma \in \Gamma$. Choose $y \in T_{0}$ and consider the horizontal circular arc

$$
\gamma_{y}=\left\{(r, \psi, \varphi)|r=| y \mid, 0 \leqq \psi \leqq \alpha, \varphi=\left(y, e_{3}\right)\right\},
$$

where $\left(y, e_{3}\right)$ is the angle between the vectors $y$ and $e_{3}$. By Hölder's inequality we obtain

$$
\begin{aligned}
1 & \leqq \int_{0}^{\alpha} \varrho r \sin \varphi d \psi=\int_{0}^{\alpha} \varrho r^{2 / 3} \sin ^{1 / 3} \varphi\left(r^{1 / 3} \sin ^{2 / 3} \varphi\right) d \psi \\
& \leqq\left(\int_{0}^{\alpha} \varrho^{3} r^{2} \sin \varphi d \psi\right)^{1 / 3}\left(\int_{0}^{\alpha} r^{1 / 2} \sin \varphi d \psi\right)^{2 / 3}
\end{aligned}
$$


Hence

and consequently

$$
\int_{0}^{\alpha} \varrho^{3} r^{2} \sin \varphi d \psi \geqq 1 /\left(\alpha^{2} r \sin ^{2} \varphi\right)
$$

$$
\begin{gathered}
\int_{D_{\alpha}\left(r_{1}, r_{2} ; \beta, \varphi_{0}\right)} Q^{3} d V=\int_{r_{1}}^{r_{2}} d r \int_{\beta}^{\varphi_{0}} d \varphi \int_{0}^{\alpha} \varrho^{3} r^{2} \sin \varphi d \psi \\
\geqq \int_{r_{1}}^{r_{2}} d r \int_{\beta}^{\varphi_{0}} \frac{d \varphi}{\alpha^{2} r \sin ^{2} \varphi}=\left(1 / \alpha^{2}\right) \frac{\sin \left(\varphi_{0}-\beta\right)}{\sin \varphi_{0} \sin \beta} \log \left(r_{2} / r_{1}\right) .
\end{gathered}
$$

From the above it follows that

$$
M(\Gamma) \geqq \frac{1}{\alpha^{2}} \cdot \frac{\sin \left(\varphi_{0}-\beta\right)}{\sin \varphi_{0} \sin \beta} \log \left(r_{2} / r_{1}\right) .
$$

Let $\Gamma_{1}$ be the arc family joining the spheres $|x|=r_{1}$ and $|x|=r_{2}$ in the set Then

$$
D_{\alpha}\left(r_{1}, r_{2} ; \beta\right)=\left\{(r, \psi, \varphi) \mid r_{1}<r<r_{2}, 0<\psi<\alpha, 0<\varphi<\beta\right\} .
$$

In particular,

$$
M\left(\Gamma_{1}\right)=\frac{\alpha}{2 \pi} \cdot \frac{\pi(2-2 \cos \beta)}{\left(\log \left(r_{2} / r_{1}\right)\right)^{2}}=\frac{\alpha(1-\cos \beta)}{\left(\log \left(r_{2} / r_{1}\right)\right)^{2}} .
$$

$$
\varliminf_{\beta \rightarrow 0} M(\Gamma)^{2} M\left(\Gamma_{1}\right) \geqq 1 / 2 \alpha^{3} .
$$

We shall next consider the $g$-images $\Gamma^{\prime}$ and $\Gamma_{1}^{\prime}$ of the families $\Gamma$ and $\Gamma_{1}$.

\section{Upper bounds for $M\left(\Gamma^{\prime}\right)$ and $M\left(\Gamma_{1}^{\prime}\right)$}

We may assume that $k\left(e_{3}\right)$ lies on the positive $x_{3}$-axis. Let

$$
\begin{aligned}
& \underline{r}_{i}=\min \left\{|g(x)||| x \mid=r_{i}, x \in \bar{D}_{\alpha}\right\} \\
& \bar{r}_{i}=\max \left\{|g(x)||| x \mid=r_{i}, x \in \bar{D}_{\alpha}\right\},
\end{aligned}
$$

where $r_{1}$ and $r_{2}$ are the radii of $D_{\alpha}\left(r_{1}, r_{2} ; \beta, \varphi_{0}\right)$ and $D_{\alpha}\left(r_{1}, r_{2} ; \beta\right)$. Furthermore we denote

and

$$
D_{\alpha}\left(\beta, \varphi_{0}\right)=\left\{(r, \psi, \varphi) \mid r>0,0<\psi<\alpha, \beta<\varphi<\varphi_{0}\right\}
$$

Define

$$
E=\left(g D_{\alpha}\left(\beta, \varphi_{0}\right)\right) \cap\left(B^{3}\left(\bar{r}_{2}\right) \backslash \overline{B^{3}\left(\underline{r}_{1}\right)}\right) .
$$

$$
\varrho(r, \psi, \varphi)=\left\{\begin{array}{l}
1 /(\pi r \sin \varphi) \text { for } \quad(r, \psi, \varphi) \in E \\
0 \text { otherwise }
\end{array}\right.
$$


We can suppose $\varphi_{0}$ so small that for the above chosen mapping $f\left(k(e), e_{3}\right)<\pi$ when $\left(e, e_{3}\right) \leqq \varphi_{0}$. Then

$$
\int_{\gamma} \varrho d s \geqq \int_{0}^{\pi}(r \sin \varphi /(\pi r \sin \varphi)) d \psi=1
$$

for all $\gamma \in \Gamma^{\prime}$, because $d s \geqq r \sin \varphi d \psi$ for every curve element. Hence

$$
M\left(\Gamma^{\prime}\right) \leqq \int_{R^{3}} \varrho^{3} d V=\int_{E} \frac{d V}{\pi^{3} r^{3} \sin ^{3} \varphi}=\frac{1}{\pi^{3}} \int_{\underline{r}_{1}}^{\bar{r}_{2}} d r \int_{E \cap S^{2}(r)} \frac{d A}{r^{3} \sin ^{3} \varphi} .
$$

Denote by $C_{r}\left(\beta, \varphi_{0}\right)$ the subset

of $S^{2}(r)$, where

$$
\left\{(r, \psi, \varphi) \mid 0 \leqq \psi \leqq \pi, \Phi \leqq \varphi \leqq \Phi^{\prime}\right\}
$$

$$
\Phi=\min _{\left(e, e_{3}\right)=\beta}\left(k(e), e_{3}\right)
$$

and $\Phi^{\prime}$ is defined such that the area of $C_{r}\left(\beta, \varphi_{0}\right)$ is equal to $m_{2}\left(E \cap S^{2}(r)\right)$. There $\Phi$ and $\Phi^{\prime}$ are independent of $r$, since $g$ maps each ray $\{t e \mid t>0\},\left(e, e_{3}\right)=\beta$, onto a ray. From the inequalities

where

$$
\int_{G(r)} \frac{d A}{\sin ^{3} \varphi} \leqq \int_{G(r)} \frac{d A}{\sin ^{3} \Phi^{\prime}}=\int_{H(r)} \frac{d A}{\sin ^{3} \Phi^{\prime}} \leqq \int_{H(r)} \frac{d A}{\sin ^{3} \varphi},
$$

it follows that

$$
\begin{aligned}
& G(r)=E \cap S^{2}(r) \backslash C_{r}\left(\beta, \varphi_{0}\right), \\
& H(r)=C_{r}\left(\beta, \varphi_{0}\right) \backslash E \cap S^{2}(r),
\end{aligned}
$$

$$
\begin{aligned}
M\left(\Gamma^{\prime}\right) & \leqq \frac{1}{\pi^{3}} \int_{\underline{r}_{1}}^{\bar{r}_{2}} d r \int_{C_{r}\left(\beta, \varphi_{0}\right)} \frac{d A}{r^{3} \sin ^{3} \varphi}=\frac{1}{\pi^{3}} \int_{\underline{r}_{1}}^{\bar{r}_{2}} \frac{d r}{r} \int_{0}^{\pi} d \psi \int_{\Phi}^{\Phi} \frac{d \varphi}{\sin ^{2} \varphi} \\
& =\frac{1}{\pi^{2}} \cdot \frac{\sin \left(\Phi^{\prime}-\Phi\right)}{\sin \Phi \sin \Phi^{\prime}} \log \left(\bar{r}_{2} / \underline{r}_{1}\right) \leqq \frac{1}{\pi^{2} \sin \Phi} \log \left(\bar{r}_{2} / \underline{r}_{1}\right) .
\end{aligned}
$$

To obtain a similar estimate for $M\left(\Gamma_{1}^{\prime}\right)$, we denote

$$
\begin{aligned}
& D_{\alpha}(\beta)=\{(r, \psi, \varphi) \mid r>0,0 \leqq \psi \leqq \alpha, 0 \leqq \varphi \leqq \beta\}, \\
& A_{r}(\beta)=\left(g D_{\alpha}(\beta)\right) \cap S^{2}(r) .
\end{aligned}
$$

Let $r_{1}$ be so small that $\bar{r}_{1}<\underline{r}_{2}$. Choose

$$
\varrho(r, \psi, \varphi)=\left\{\begin{array}{l}
1 /\left(r \log \left(\underline{r}_{2} / \bar{r}_{1}\right)\right) \text { for } \quad(r, \psi, \varphi) \in g D_{\alpha}(\beta) \cap\left(\overline{B^{3}\left(\underline{r}_{2}\right)} \backslash B^{3}\left(\bar{r}_{1}\right)\right) \\
0 \quad \text { otherwise. }
\end{array}\right.
$$


Then

$$
\int_{\gamma} \varrho d s \geqq \int_{\bar{r}_{1}}^{\underline{r}_{2}} \frac{d r}{r \log \left(\underline{r}_{2} / \bar{r}_{1}\right)}=1
$$

for every $\gamma \in \Gamma_{1}^{\prime}$, i.e. $\varrho \in F\left(\Gamma_{1}^{\prime}\right)$, and consequently

$$
M\left(\Gamma_{1}^{\prime}\right) \leqq \int_{R^{3}} \varrho^{3} d V=\int_{\bar{r}_{1}}^{\underline{r}_{2}} d r \int_{A_{r}(\beta)} \frac{d A}{r^{3} \log ^{3}\left(\underline{r}_{2} / \bar{r}_{1}\right)}=\left(1 / \log ^{3}\left(\underline{r}_{2} / \bar{r}_{1}\right)\right) \int_{\bar{r}_{1}}^{\underline{r}_{2}} \frac{m_{2}\left(A_{r}(\beta)\right)}{r^{2}} \frac{d r}{r}
$$

If we denote

then

$$
\bar{\Phi}=\max _{x \in A_{r}(\beta)}\left(x, e_{3}\right)=\max _{e \in D_{\alpha}(\beta)}\left(k(e), e_{3}\right),
$$

(3) $M\left(\Gamma_{1}^{\prime}\right) \leqq\left(\log \left(\underline{r}_{2} / \bar{r}_{1}\right)\right)^{-3} \int_{\bar{r}_{1}}^{\underline{r}_{2}} \pi(1-\cos \bar{\Phi}) d r / r=2 \pi(\sin (\bar{\Phi} / 2))^{2}\left(\log \left(\underline{r}_{2} / \bar{r}_{1}\right)\right)^{-2}$.

\section{Proof of Theorem 1}

Keeping $r_{1}$ and $r_{2}$ fixed we let now $\beta \rightarrow 0$. By the condition $\mathrm{B}$, the estimates (2) and (3) imply

$$
\varlimsup_{\beta \rightarrow 0} M\left(\Gamma^{\prime}\right)^{2} M\left(\Gamma_{1}^{\prime}\right) \leqq \frac{1}{2 \pi^{3}}\left(\log \left(\bar{r}_{2} / \underline{r}_{1}\right) / \log \left(\underline{r}_{2} / \bar{r}_{1}\right)\right)^{2} .
$$

Since the extended mapping $\hat{g}: R^{3} \rightarrow R^{3}$ is quasiconformal, $\varlimsup_{r_{1} \rightarrow 0}\left(\bar{r}_{1} / \underline{r}_{1}\right)$ is finite and with a fixed $r_{2}$

$$
\lim _{r_{1} \rightarrow 0}\left(\log \left(\bar{r}_{2} / \underline{r}_{1}\right) / \log \left(\underline{r}_{2} / \bar{r}_{1}\right)\right)=1
$$

The above estimate (4) is valid for every pair $r_{1}, r_{2}>0$ with small $r_{1}$, and letting $r_{1} \rightarrow 0$ with fixed $r_{2}$ we obtain

$$
\varlimsup_{r_{1} \rightarrow 0}\left(\varlimsup_{\beta \rightarrow 0} M\left(\Gamma^{\prime}\right)^{2} M\left(\Gamma_{1}^{\prime}\right)\right) \leqq 1 / 2 \pi^{3} .
$$

Thus the inequalities $M(\Gamma) \leqq K_{o}(g) M\left(\Gamma^{\prime}\right)$ and $M\left(\Gamma_{1}\right) \leqq K_{o}(g) M\left(\Gamma_{1}^{\prime}\right)$ imply

$$
K_{o}(g) \geqq \pi / \alpha .
$$

Because $\varepsilon>0$ in (1) is arbitrary, it follows

$$
K_{o}\left(D_{\alpha}, D_{\pi} ; W\right) \geqq \pi / \alpha .
$$

The example $f \in W, f(r, \varphi, z)=(r, \pi \varphi / \alpha, \pi z / \alpha)$, given in cylindrical coordinates, shows that

Thus the theorem is proved.

$$
K_{O}\left(D_{\alpha}, D_{\pi} ; W\right) \leqq \pi / \alpha .
$$




\title{
References
}

[1] Agard, S.: Angles and quasiconformal mappings in space. - J. Analyse Math. 22, 1969, 177200.

[2] Gehring, F. W., and J. VÄISÄLÄ: The coefficients of quasiconformality of domains in space. - Acta Math. 114, 1965, 1-70.

[3] VÄISÄLÄ, J.: Lectures on $n$-dimensional quasiconformal mappings. - Lecture Notes in Mathematics 229, Springer-Verlag, Berlin - Heidelberg - New York, 1971.

\author{
University of Helsinki \\ Department of Mathematics \\ SF-00100 Helsinki 10 \\ Finland
}

Received 3 December 1976 